Research Paper

\title{
Exploring brusatol as a new anti-pancreatic cancer adjuvant: biological evaluation and mechanistic studies
}

\author{
Zheng Lu ${ }^{1,2,4}$, Zheng-Quan Lai ${ }^{1}$, Albert W.N. Leung ${ }^{1}$, Po Sing Leung ${ }^{3}$, Zhao-Shen Li ${ }^{2}$ \\ and Zhi-Xiu Lin ${ }^{1}$ \\ ${ }^{1}$ School of Chinese Medicine, Faculty of Medicine, The Chinese University of Hong Kong, Shatin, China \\ ${ }^{2}$ Department of Gastroenterology, Changhai Hospital, The Second Military Medical University, Shanghai, China \\ ${ }^{3}$ School of Biomedical Sciences, Faculty of Medicine, The Chinese University of Hong Kong, Shatin, China \\ ${ }^{4}$ Liver Cirrhosis Diagnosis and Treatment Center, Beijing 302 Hospital, Beijing, China \\ Correspondence to: Zhi-Xiu Lin, email: linzx@cuhk.edu.hk \\ Keywords: pancreatic cancer, brusatol, gemcitabine, 5-fluorouracil, combination therapy \\ Received: September 24, $2016 \quad$ Accepted: April 17, $2017 \quad$ Published: May 10, 2017 \\ Copyright: Lu et al. This is an open-access article distributed under the terms of the Creative Commons Attribution License 3.0 \\ (CC BY 3.0), which permits unrestricted use, distribution, and reproduction in any medium, provided the original author and source \\ are credited.
}

\section{ABSTRACT}

Pancreatic cancer is highly resistant to chemotherapeutic agents and is known to have a poor prognosis. The development of new therapeutic entities is badly needed for this deadly malignancy. In this study, we demonstrated for the first time that brusatol, a natural quassinoid isolated from a Chinese herbal medicine named Bruceae Fructus, possessed potent cytotoxic effect against different pancreatic adenocarcinoma cell lines. Its anti-pancreatic cancer effect was comparable to that of the first-line chemotherapeutic agents such as gemcitabine and 5-fluorouracil, with a more favorable safety profile. In addition, brusatol showed a synergistic anti-proliferative effect toward PANC-1 and Capan-2 cell lines when combined with gemcitabine or 5-fluorouracil. The results of flow cytometry suggested that brusatol combination treatment with gemcitabine or 5-fluorouracil was able to cause cell cycle arrest at G2/M phase, and accentuate apoptosis in PANC-1 cells. Moreover, brusatol deactivated gemcitabine/5fluorouracil-induced NF-KB activation. Western blot analysis and qRT-PCR results showed that brusatol significantly down-regulated the expression of vimentin and Twist, and markedly stimulated the expression of E-cadherin, the key regulatory factors of the epithelial-mesenchymal transition process. Furthermore, treatment with combination of brusatol and gemcitabine or 5-fluorouracil significantly reduced in vivo tumor growth when compared with treatment of either brusatol or gemcitabine/5fluorouracil alone. Taken together, these results have amply demonstrated that brusatol is a potent anti-pancreatic cancer natural compound, and the synergistic anti-pancreatic cancer effects of brusatol and gemcitabine/5-fluorouracil observed both in vitro and in vivo are associated with the suppression of epithelial-mesenchymal transition process, indicating that brusatol is a promising adjunct to the current chemotherapeutic regimen.

\section{INTRODUCTION}

Pancreatic cancer (PanCa), one of the most deadly human malignancies, has a 5-year survival rate of less than $1 \%$. PanCa is the $4^{\text {th }}$ most common cause of the cancerrelated death in the USA [1], where in 2014, about 46,420 people were diagnosed with $\mathrm{PanCa}$ and approximately
39,590 people died of this disease [2]. In China, the past two decades have witnessed a 6-time increase in the incidence rate of PanCa, possibly owing to the increasing prevalence of Western diets-induced obesity. Today, PanCa has become the $6^{\text {th }}$ leading cause of cancer-related mortality in China [3]. Currently, pharmacological treatment using gemcitabine (GEM) and 5-fluorouracil (5-FU) is among 
the most common strategies for the treatment of advanced and unresectable PanCa. However, the median survival time is still less than 6 months for patients on these treatments [4], due mainly to drug resistance [5]. To overcome chemo-resistance, combination chemotherapy strategies are usually adopted to achieve better cancer cell killing with fewer systemic toxicity [6]. To enhance the efficacy of current chemotherapeutic agents such as GEM and 5-FU for PanCa, new adjuvants which can circumvent GEM/5-FU-associated chemo-resistance are badly needed.

In recent years, herbal medicines or natural compounds, either used alone or combined with conventional chemotherapeutic agents, have been shown to have beneficial effects on diverse cancers [7]. In our previous work, we found that the alcoholic extract of Bruceae Fructus (Ya-Dan-Zi in Chinese), a Chinese medicinal herb commonly used for the treatment of cancer, possessed significant cytotoxicity against several PanCa cell lines [8]. Brucein D, a quassinoid found in abundance in this herb, has been shown to inhibit the activity of NF- $\kappa \mathrm{B}$ and suppress the growth of several PanCa cell lines via inducing cellular apoptosis without causing overt organ toxicity $[9,10]$. Brusatol (BR), a natural quassinoid diterpenoid isolated from Bruceae Fructus, exhibited the most potent in vitro antipancreatic tumor action among all the isolated quassinoids [11]. Furthermore, it was reported that brusatol acted as a unique inhibitor of the Nrf2 pathway that sensitized various cancer cells and A549 xenografts to chemotherapeutic drugs, suggesting brusatol might be a promising candidate for combating chemo-resistance and has the potential to be developed into an adjuvant chemotherapeutic agent [12]. However, the chemosensitizing effect of brusatol on PanCa has not been explored.

It has been known that epithelial-mesenchymal transition (EMT) involving key regulators such as Twist and E-cadherin, is an important mechanism underlying chemotherapy resistance $[13,14]$. Targeted inhibition of the EMT process without eliciting systemic toxicity using combination chemotherapeutic agents may lead to better tumor cell killing in PanCa. Based on the antiPanCa and chemosensitizing effect of brusatol, we hypothesized that brusatol could sensitize the current first-line chemotherapeutic agents GEM and 5-FU to PanCa via inhibition on the EMT process. This work was therefore initiated to explore the potential of brusatol as a novel anti-PanCa adjuvant for GEM and 5-FU using different PanCa cell lines in vitro, and in vivo via orthotopic xenotransplantation PanCa mice model. Moreover, the underlying molecular mechanisms were delineated. Our results indicated that brusatol deactivated $\mathrm{NF}-\kappa \mathrm{B}$ activation and arrested PanCa cell growth at least in part via inhibition of Twist and stimulation of E-cadherin expression. The synergistic inhibition by brusatol and GEM or 5-FU observed both in vitro and in vivo suggested that brusatol may be a promising adjunct to current chemotherapeutic regimens.

\section{RESULTS}

\section{Brusatol inhibits proliferation and potentiates the inhibitory effects of chemotherapeutic agents in PanCa cells}

The cytotoxic effects of brusatol, GEM and 5-FU on the cell viability of human PanCa cell lines PANC-1, Capan-1, Capan-2, SW1990, and the non-tumorigenic human gastric cell line (GES-1) are shown in Figure 1A-1C. The results showed that brusatol markedly suppressed the cell proliferation of all the four tested PanCa cell lines in a dose- and time-dependent manner, with $\mathrm{IC}_{50}$ values in the range of $0.33-8.47 \mu \mathrm{g} / \mathrm{mL}$. The potency of brusatol was significantly higher than those of GEM ( $\left.\mathrm{IC}_{50}: 2.83-78.78 \mu \mathrm{g} / \mathrm{mL}\right)$ and 5-FU $\left(\mathrm{IC}_{50}: 1.48\right.$ $85.11 \mu \mathrm{g} / \mathrm{mL}$ ). However, brusatol only exerted mild cytotoxicity on GES-1 cells, with $\mathrm{IC}_{50}$ value $>68.90 \mu \mathrm{g} /$ $\mathrm{mL}$. In contrast, GEM and 5-FU were more toxic to GES1 cells, with $\mathrm{IC}_{50}$ values being $5.92 \mu \mathrm{g} / \mathrm{mL}$ and $12.34 \mu \mathrm{g} /$ $\mathrm{mL}$, respectively. These results unambiguously indicated that brusatol possessed potent in vitro anti-PanCa effect.

For single-agent treatment, GEM or 5-FU $(0.1,1$, $5,10,100,500 \mu \mathrm{g} / \mathrm{mL}$ ) alone produced a dose-dependent inhibition on the growth of PANC-1 and Capan-2 cells when cultured for $72 \mathrm{~h}$. When GEM or 5-FU was combined with brusatol at a constant concentration ratio of $10: 1$, by adding $0.01,0.1,0.5,1,10$ and $50 \mu \mathrm{g} / \mathrm{mL}$ brusatol, respectively, the cell growth inhibition was greatly enhanced (Figure 1D). The combinationindex (CI) is a mathematical method commonly used to measure the pharmacological interaction of two drugs [15-18]. Isobologram analysis showed that the CI for every combination treatment was $<1$ (Figure $1 \mathrm{E}$ and Supplementary Table 1), indicating significant synergistic effects of these combination treatments. It is clear from the results that combination of GEM or 5-FU with lower doses of brusatol elicited significantly greater inhibition on the cancer cell growth than either agent alone.

Since brusatol showed a synergistic antiproliferative effect when combined with GEM or 5-FU in PANC-1 and Capan-2 cell lines, and PANC-1 and Capan-2 cells were more sensitive to the combination treatment and relatively easier to culture, they were therefore used for the subsequent mechanistic studies.

\section{Brusatol induces apoptosis and causes G2/M cell cycle arrest in PanCa cells}

Quantification of DNA fragmentation in PANC1 and Capan-2 cells was performed using Cell Death Detection ELISA ${ }^{\text {pLUS }}$ Kit. Cells were treated with DMEM medium (control), GEM (10 $\mu \mathrm{g} / \mathrm{mL}), 5$-FU $(10 \mu \mathrm{g} / \mathrm{mL})$, brusatol $(2 \mu \mathrm{g} / \mathrm{mL})$ or combination treatment for $48 \mathrm{~h}$. The results revealed that brusatol caused significant DNA fragmentation after exposure for $48 \mathrm{~h}$. Noticeably, brusatol 
combined with GEM or 5-FU induced more cellular apoptosis than the GEM or 5-FU alone group (Figure 2A). Quantitative flow cytometric analysis showed that brusatol exerted a dose- and time-dependent apoptogenic effect on PANC-1 cells (Figure 2B). At 1 and $2 \mu \mathrm{g} / \mathrm{mL}$, brusatol caused $10.9 \%$ and $15.5 \%$ of the cancer cells to undergo apoptosis within $24 \mathrm{~h}$, respectively. Extending the treatment time to $48 \mathrm{~h}$ resulted in a marked augmentation of cellular apoptosis, with $36.8 \%$ and $48.1 \%$ of cellular apoptosis after 1 and $2 \mu \mathrm{g} / \mathrm{mL}$ brusatol treatments, respectively. In stark contrast, non-treated cells showed normal cell viability without significant cell death.

To further elucidate the mechanism of growth inhibition and whether the cell cycle change upon brusatol monotherapy and combination treatment, PANC-1 cells were treated with $2 \mu \mathrm{g} / \mathrm{mL}$ brusatol for 24,48 and $72 \mathrm{~h}$, and exposed to brusatol alone and in combination with GEM or 5-FU for $48 \mathrm{~h}$. The results indicated that brusatol alone induced a time-dependent $\mathrm{G} 2 / \mathrm{M}$ arrest (Figure 2C), and brusatol combined with GEM or 5-FU also resulted in a pronounced accumulation of cells in G2/M (Figure 2D).

\section{Brusatol treatment decreases the expression of anti-apoptotic protein and deactivates chemotherapeutic agents-induced NF-кB activation}

As shown in Figure $3 \mathrm{~A}$ and $3 \mathrm{C}$, immunoblotting results clearly showed the down-regulation of NF$\kappa \mathrm{B}$ p65 in PanCa cells after exposure to brusatol, and the combined treatment with chemotherapeutic agents significantly augmented the brusatol-mediated inhibition of NF- $\kappa \mathrm{B}$ p65. In addition, brusatol down-regulated the expression of two anti-apoptotic proteins $\mathrm{Bcl}-\mathrm{xL}$ and PCNA in both PANC-1 and Capan-2 cells. Combination treatment of brusatol with GEM or 5-FU also markedly attenuated the expression of Bcl-xL and PCNA (Figure $3 \mathrm{~B}, 3 \mathrm{D}$ and $3 \mathrm{E}$ ). The immunoblotting results are strongly indicative that brusatol could suppress the expression of anti-apoptotic proteins in favor of promoting cellular apoptosis. These findings were congruent with cell growth inhibition as observed in MTT assay, amply vindicating that the greater cell growth inhibition observed with the
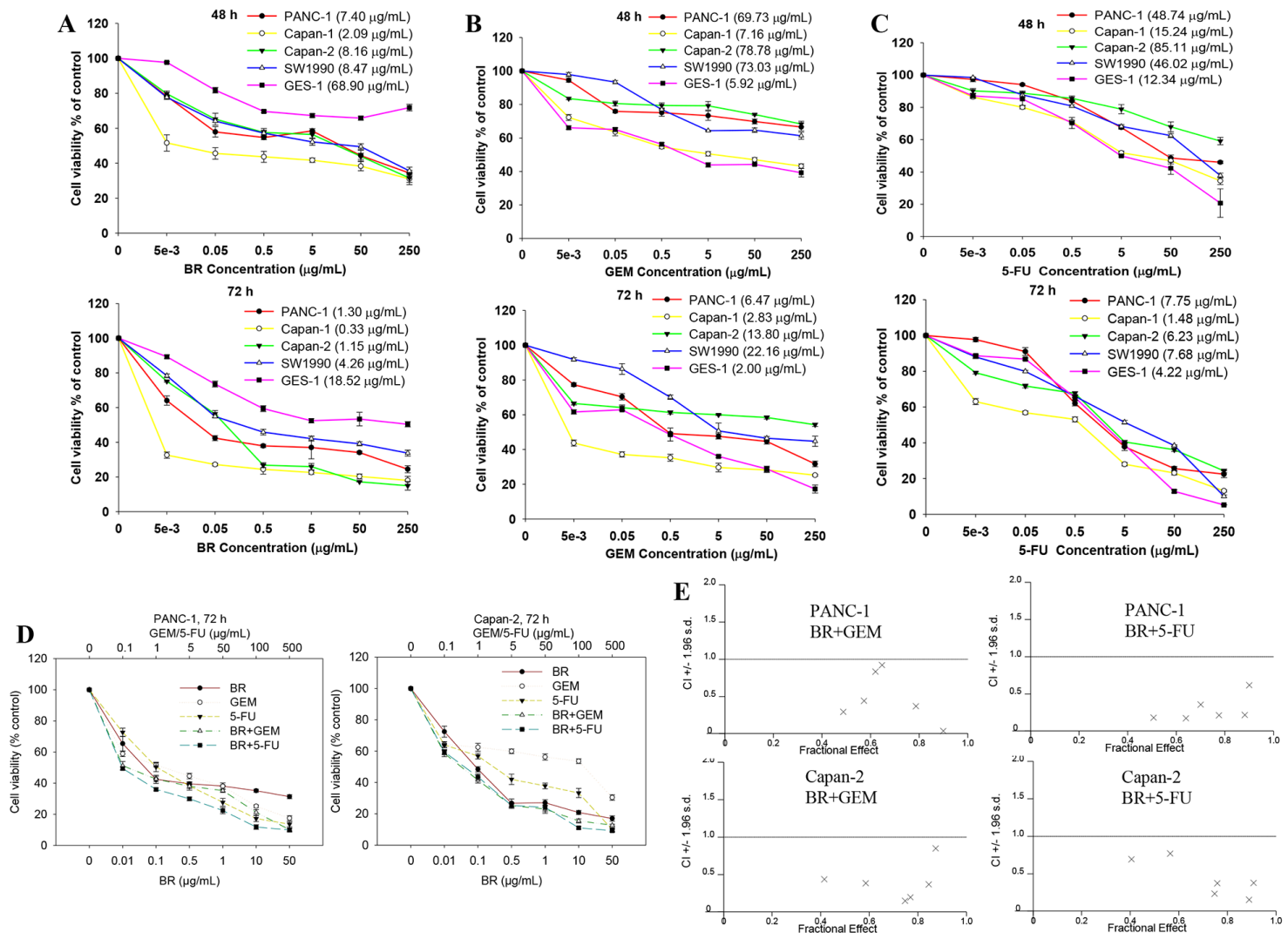

Figure 1: Brusatol (BR) inhibits proliferation and potentiates the inhibitory effects of chemotherapeutic agents in PanCa cells. (A-C) Cytotoxic effects of brusatol (A), GEM (B) and 5-FU (C) on the growth of human PanCa cell lines, and non-tumorigenic human gastric cell line (GES-1). $\mathrm{IC}_{50}$ values were shown in parentheses immediately after the names of the cell lines in the graphic legends. (D) Cell growth inhibition elicited by GEM, 5-FU and the combination with brusatol in PANC-1 and Capan-2 cells as measured by MTT assay. (E) Fa-CI plots for combination treatment with brusatol and GEM or 5-FU in PANC-1 and Capan-2 cells. Combination index (CI) is a quantitative measurement of the degree of drug interaction. $\mathrm{CI}<1$ indicates synergism, $\mathrm{CI}=1$ indicates additive effect, while $\mathrm{CI}>1$ signifies antagonism. $\times$ represents that CI values were generated over a range of $40 \%-95 \%$ growth inhibitory effects. 
combination treatment was closely associated with the induction of cellular apoptosis.

\section{Effects of brusatol on the EMT process in PanCa cells}

EMT is an important mechanism associated with chemoresistance. In the present work, the expression of E-cadherin, vimentin and Twist, three characteristic factors of the EMT process, was detected by Western blotting after brusatol alone or combination treatment for $48 \mathrm{~h}$. The results showed that brusatol markedly increased the E-cadherin expression, while significantly decreased vimentin expression. As shown in Figure $4 \mathrm{~A}$ and $4 \mathrm{~B}$, brusatol combined with chemotherapeutic agents induced stronger E-cadherin protein expression in PANC-1 cells, with 3.3-fold over that of GEM and 2.7fold 5-FU, respectively. In contrast, the expression of vimentin (Figure 4A and 4C) and Twist (Figure 4A and 4D) decreased significantly after combination treatment when compared with the control.
Effects of brusatol on E-cadherin and Twist expression in PANC-1 and Capan-2 cells were further analyzed by real-time PCR. The results revealed the significantly increased E-cadherin mRNA expression and decreased Twist expression in both the brusatol monotherapy and combination treatments, as compared with the untreated control (Figure 4E and 4F). The results implied that brusatol alone or in combination with chemotherapeutic agents could increase the expression of E-cadherin, while suppress the expression of Twist and vimentin, thus inhibiting the EMT process, and ultimately leading to the chemosensitizing effect of brusatol.

\section{Brusatol and its combination with chemotherapeutic agents significantly inhibits the tumor growth in human pancreatic orthotopic xenograft tumor mouse model}

Based on the promising in vitro results concerning the anti-PanCa effect of brusatol and its combination with GEM or 5-FU, we undertook the in vivo studies to

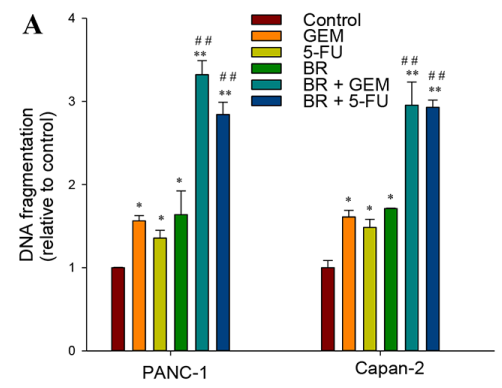

C
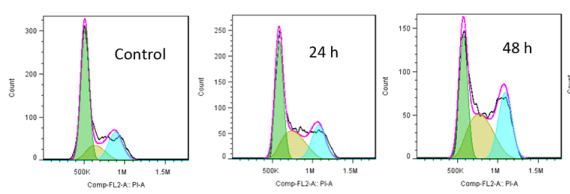

D
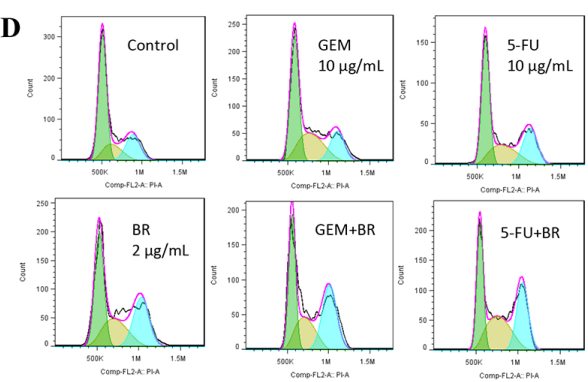
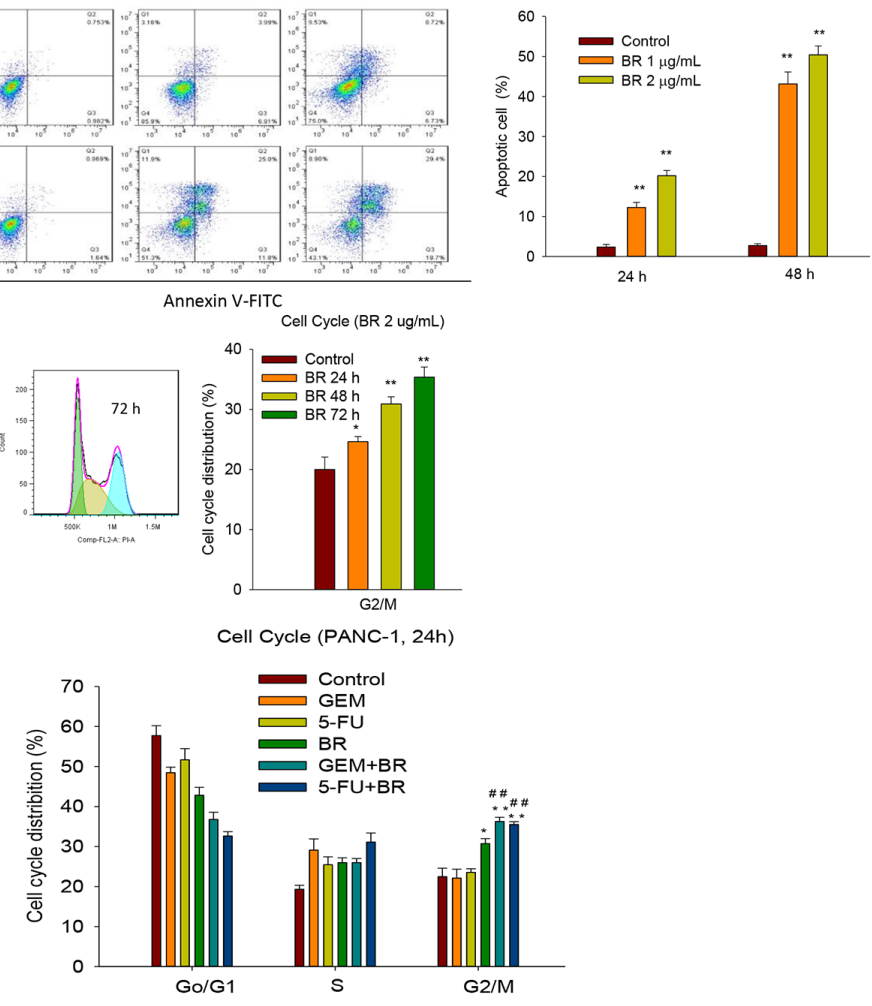

Figure 2: Brusatol induces apoptosis and causes G2/M cell cycle arrest in PanCa cells. (A) Quantitative measurement of

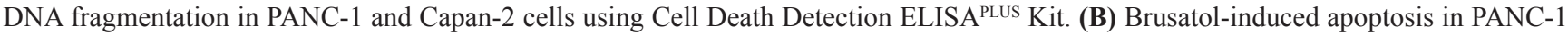
cells detected by Annexin V-PI stain assay. Criteria were set to distinguish between viable (bottom left), early apoptotic (bottom right), late apoptotic (top right) and necrotic (top left) cells. (C) Brusatol time-dependently arrested cell cycle at the G2/M phase as assessed by PI staining. (D) Combination treatment with brusatol and GEM or 5-FU significantly arrested cell cycle at the G2/M phase as assessed by PI staining at $24 \mathrm{~h}$. The data in A-D are represented as the mean $\pm \mathrm{SEM} ;{ }^{*} P<0.05$, $* * P<0.01$ vs control and $\# P<0.05$, \#\#P<0.01 vs chemotherapeutic agent treatment alone. All data are representative of three independent experiments. 
investigate whether brusatol alone, or in combination with chemotherapeutic agents, could inhibit the growth of pancreatic tumor in an orthotopic animal model.

The lentivirus expressing EGFP and luciferase were used to infect PANC-1 and Capan-2 cells. We observed under a fluorescent microscope for the expression of EGFP at different time-points after infecting the cells with lentivirus and then calculated the infection efficiency. When the multiplicity of infection (MOI) was 100 , the infection efficiency of lentivirus was above $80 \%$ after incubation for $72 \mathrm{~h}$ in PANC-1 and Capan-2 cells. Following 2-week screening by puromycin, the infection efficiency was maintained stably in the range of 80 $90 \%$ (Figure $5 \mathrm{~A}$ ). The results of luciferase activity assay showed that lentivirus could steadily infect PANC-1 and Capan-2 cells and the infection efficiency was satisfactory. In addition, there was no significant difference in morphology, growth rate and tumor formation rate between parent and CMV-EGFP-linker-Luc-transfected cells (data not shown). Hence, the cell lines expressing both EGFP and luciferase could serve as a promising tool for real-time monitoring of tumor growth in vivo.

For the construction of orthotopic PanCa mouse model, PANC-1 and Capan-2 cells (stably transfected with EGFP and luciferase) were injected into the pancreas as described in the Methods. The experimental protocol is depicted in Figure 5B. As shown in Figure 5C and 5G, the luciferase fluorescence made possible the real-time and sequential whole-body imaging of tumors. Moreover, noninvasive quantitative measurements of the externally visible fluorescent area enabled the construction of the in vivo tumor growth curves (Figure 5D and 5H). Small primary tumor lesions on day 7 after transplantation were observed in all mice by the real-time whole-body imaging. The differences in imaging were not significant on day 14. However, imaging conducted on days 21, 28 and 35 confirmed the significant growth of primary tumor in the control group. While 1 or $2 \mathrm{mg} / \mathrm{kg}$ brusatol group, and its combination with GEM or 5-FU groups showed a marked reduction in the tumor growth in a dose-dependent manner when compared with the control. The size of xenograft tumors formed in mice of $2 \mathrm{mg} / \mathrm{kg}$ group was markedly smaller than that of the $1 \mathrm{mg} / \mathrm{kg}$ brusatol-treated mice. On days 28 and 35, in both PANC-1 and Capan-2 orthotopic xenograft tumor models, brusatol (1 or $2 \mathrm{mg}$ / $\mathrm{kg}$ ), and its combination with GEM or 5-FU were shown to significantly inhibit the tumor size as compared with the corresponding control (Figure 5C, 5D, 5G and 5H).

Figure 5E and 5I showed the effects of brusatol and chemotherapeutic agents on the tumor weight and volume
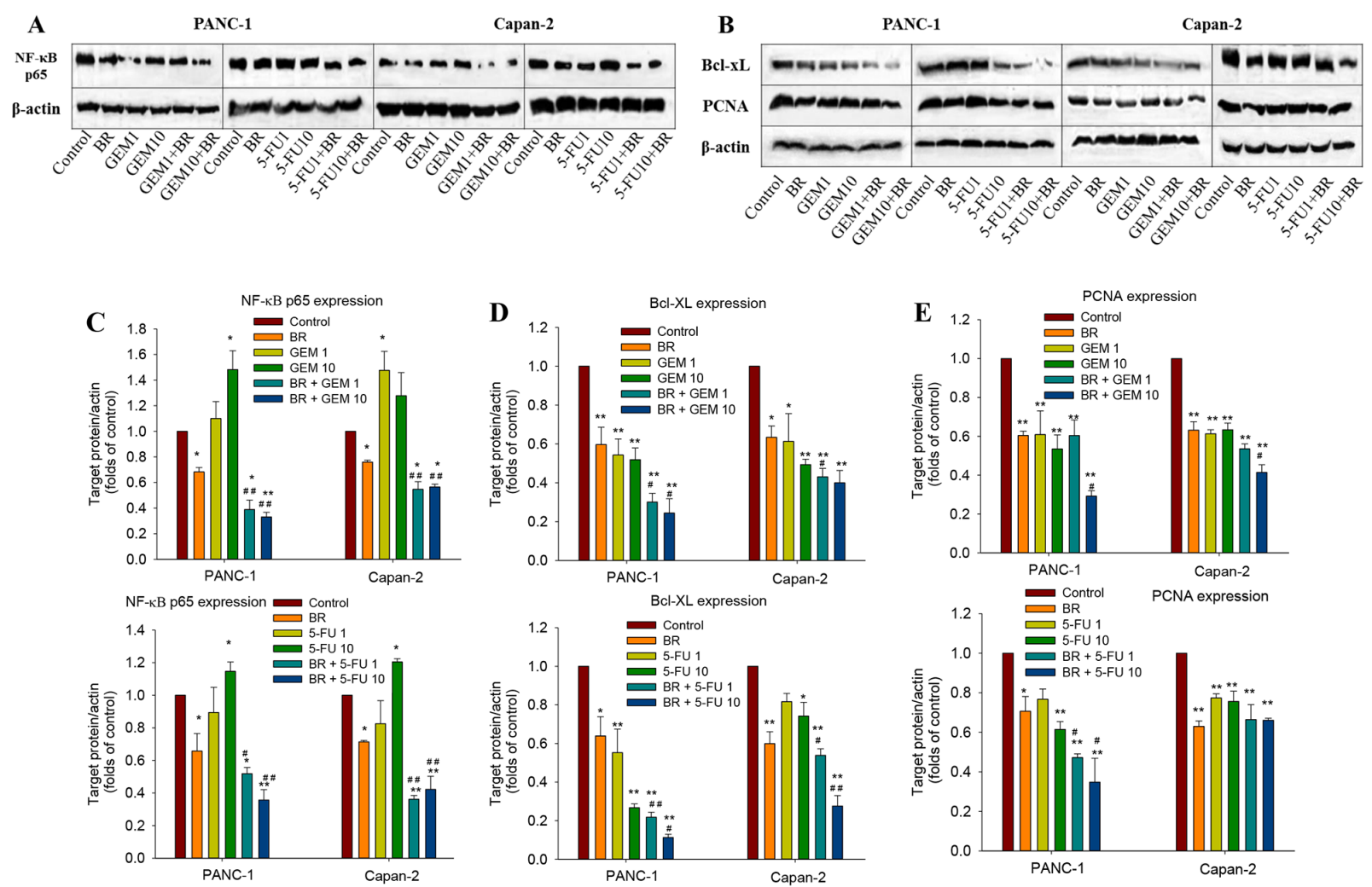

Figure 3: Brusatol deactivates chemotherapeutic agents-induced NF- $\kappa$ Bactivation and inhibits the anti-apoptotic protein in PanCa cells. (A-B) Western blot analysis of the expression of NF- $\kappa B$ p65, Bcl-xL and PCNA in PANC-1 and Capan-2 cells. (C-E) Quantitative analysis of Western blot band intensities. The data in C-E are represented as the mean \pm SEM; ${ }^{*} P<0.05, * * P<0.01$ vs control and $\# P<0.05, \# \# P<0.01$ vs chemotherapeutic agent treatment alone. All data are representative of three independent experiments. 
as measured at the end of the experiment by autopsy. Results indicated that brusatol, GEM, 5-FU and their combinations all significantly reduced the tumor volume and weight as compared with the corresponding control in both orthotopic xenograft tumor models (Figure 5E, 5F, 5I and 5J). Brusatol at $1 \mathrm{mg} / \mathrm{kg}$ was also found to exhibit significant anti-tumor activity albeit the effect was less potent when compared with that of $2 \mathrm{mg} / \mathrm{kg}$ brusatol. The combined treatment of brusatol at $2 \mathrm{mg} / \mathrm{kg}$ with chemotherapeutic agents significantly reduced the tumor volume and weight, and the tumor reduction was more pronounced than either agent alone.

\section{In vivo toxicity test of brusatol}

To evaluate the potential toxicity of brusatol, acute toxicity assessment was performed.Nude mice treated with brusatol intraperitoneally at the high dose $(2 \mathrm{mg} / \mathrm{kg})$ for 28 consecutive days did not show any treatmentrelated side effects and signs of toxicity. The body weight of the brusatol-treated and combination-treated nude mice showed no significant changes when compared to that of the GEM/5-FU alone treatment. A larger accrual in the body weights of the control mice observed might be caused by more rapid tumor growth in control group (Figure 6A). In addition, no significant differences were observed in the levels of plasma enzymes including ALT, AST, LDH, CK and Cr (Figure 6B) between the treatment groups and the control. Furthermore, brusatol induced no treatment-related abnormality concerning the gross anatomy and histological morphology. The results indicated that brusatol at the high dose of $2 \mathrm{mg} / \mathrm{kg}$ exerted no overt toxicity to the liver, heart and kidney tissues in the tumor-bearing mice.

\section{Expression of E-cadherin and Twist in the orthotopic xenograft tumor tissues}

The Twist and E-cadherin expression levels in pancreatic tumor tissues were measured. We found that both brusatol and 5-FU alone groups upregulated the expression of E-cadherin in PANC-1 and Capan-2
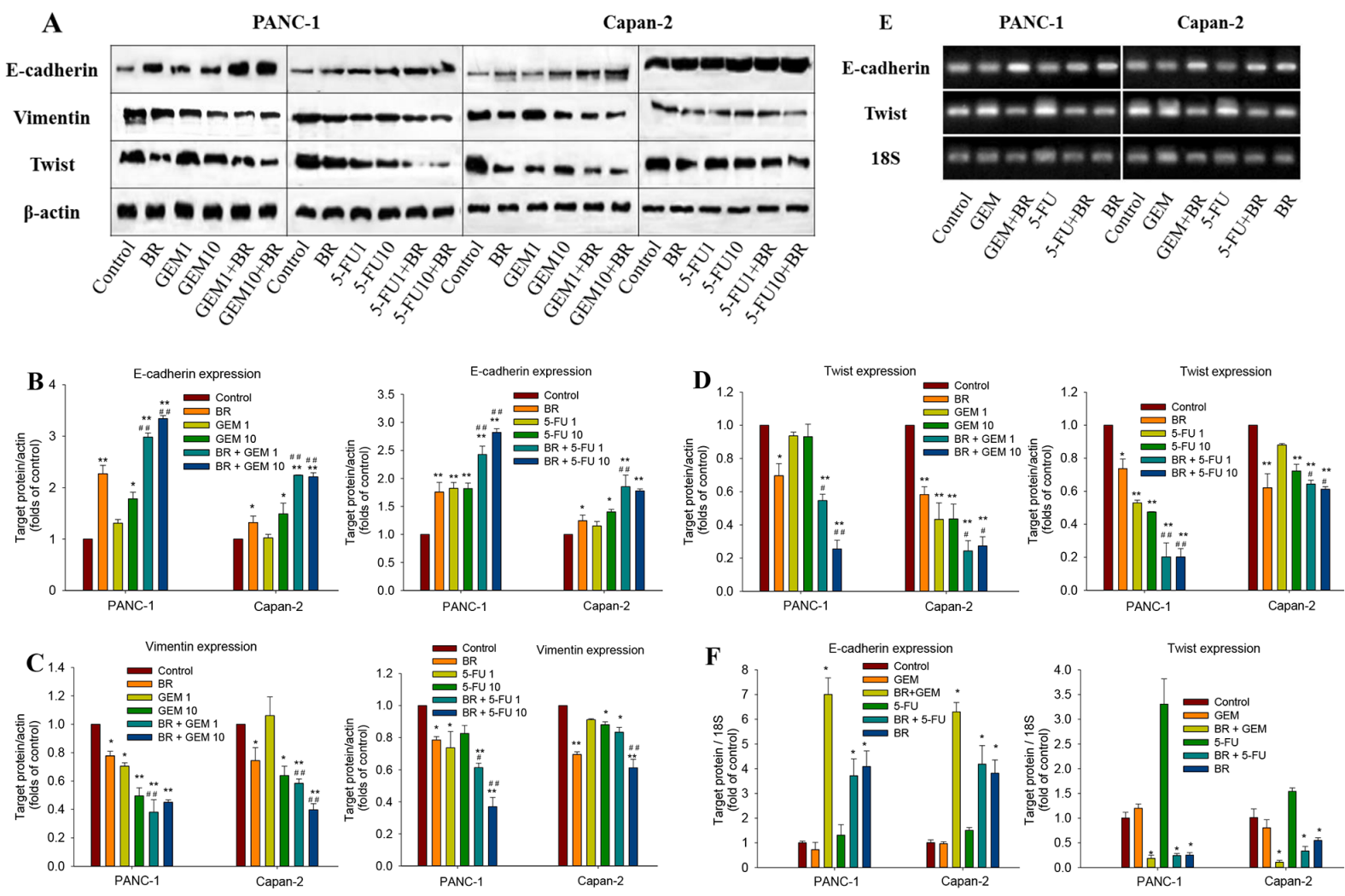

Figure 4: Effects of brusatol on the EMT process in PanCa cells. (A) Western blot analysis of the expression of key regulatory factors (E-cadherin, vimentin, and Twist) of the EMT process in PANC-1 and Capan-2 cells. (B-D) Quantitative analyses of the Western blot band intensities. (E) qRT-PCR analysis of the mRNA expression of E-cadherin and Twist. The electrophoresis of RT-PCR product was amplified by 18S, E-cadherin and Twist primers. (F) Brusatol significantly increased E-cadherin mRNA expression and decreased Twist mRNA expression alone and in combination with GEM or 5-FU in both PANC-1 and Capan-2 cells. Expression of E-cadherin and Twist was normalized against the housekeeping gene $18 \mathrm{~S}$. The data in $\mathrm{B}-\mathrm{D}$ and $\mathrm{F}$ are represented as the mean $\pm \mathrm{SEM} ; * P<0.05$, ** $P<0.01 v s$ control, and $\# P<0.05, \# \# P<0.01$ vs chemotherapeutic agent treatment alone. All data are representative of three independent experiments. 
orthotopic xenograft tumor tissues (Figure 7A-7D). The result confirmed our in vitro data that brusatol treatment upregulated the E-cadherin levels in PanCa cells. Similarly, we found that brusatol alone decreased the Twist expression, as compared with the control group. The differences in the expression of E-cadherin and Twist between different brusatol groups and the control group were statistically significant. In addition, in both PANC-1 and Capan-2 orthotopic xenograft tumor tissues, brusatol combined with GEM or 5-FU group showed stronger staining for E-cadherin, and weaker Twist staining when compared with the chemotherapeutic agent alone and the control group, and the combination treatment was observed to produce the most obvious effect $(P<0.01)$.

\section{DISCUSSION}

Brusatol, a quassinoid found in abundance in Bruceae Fructus, was believed to be one of the major active principles responsible for the anticancer effect of Bruceae Fructus. In the present study, pioneering effort was devoted to investigate the in vitro and in vivo chemosensitizing effect of brusatol toward GEM and 5-FU, and to unravel the potential underlying molecular mechanisms.

We have demonstrated in the present study that brusatol significantly inhibited the proliferation of human PanCa cells in a dose- and time- dependent manner, and brusatol at low concentration was able to significantly
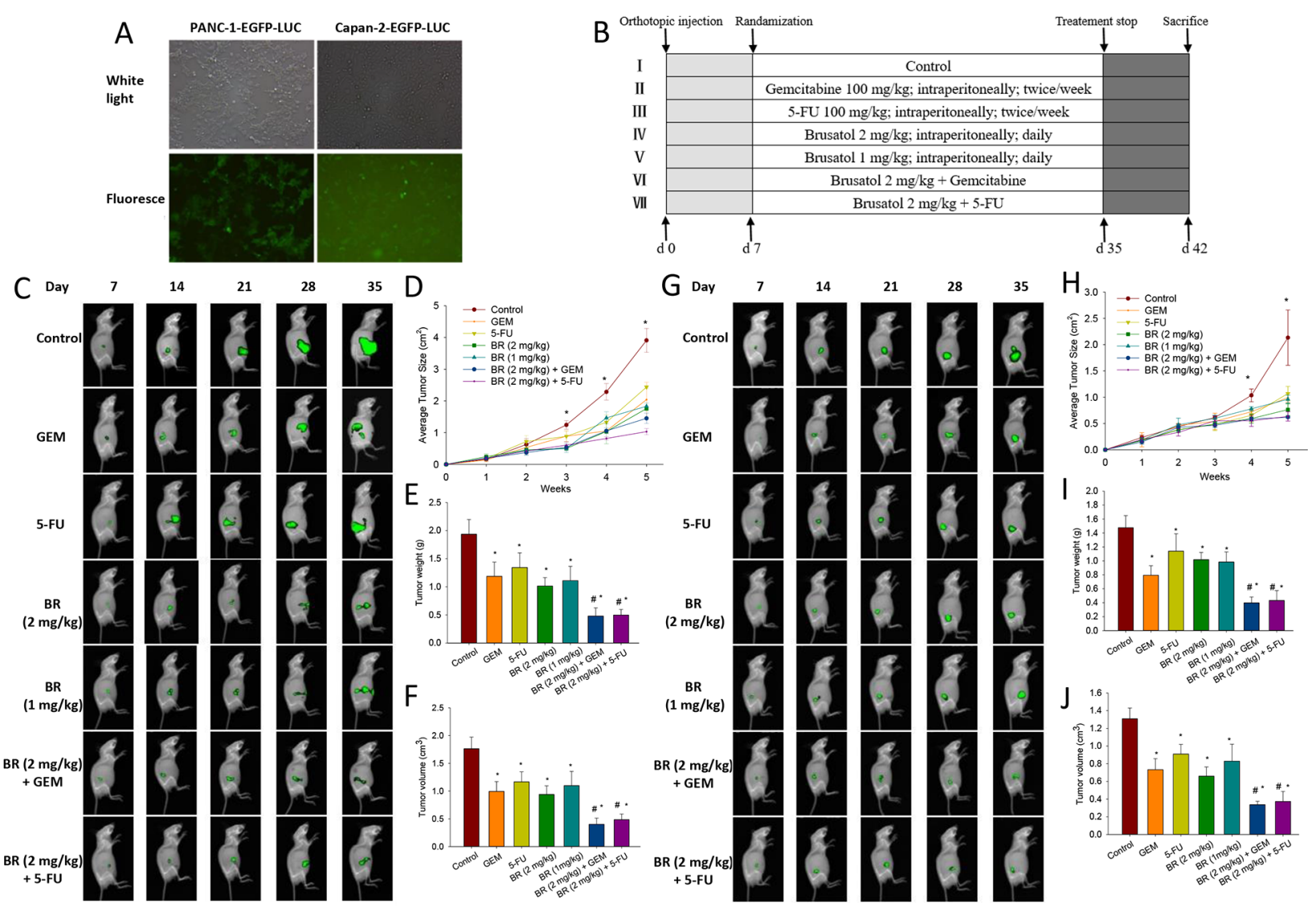

Figure 5: Brusatol and its combination with chemotherapeutic agents significantly inhibit the tumor growth in human pancreatic orthotopic xenograft mouse model. (A) Expression of EGFP was observed by fluorescent microscopy. Lentivirus vector transfection produced up to $80 \%$ EGFP-positive cells after 14 days as screened by puromycin. (B) Schematic presentation of the experimental protocol described in the Materials and Methods. (C-F) Brusatol alone or in combination with chemotherapeutic agents markedly inhibited the tumor growth in PANC-1 orthotopic xenograft mouse model. Whole-body imaging, real-time analysis of pancreatic tumor growth was performed in live, anesthetized mice every week. Panels depict a representative mouse from each group (C). Quantification of orthotopic xenograft tumor luciferase fluorescence enabled real-time determination and comparison of tumor load during the course of treatment, thereby permitting real-time comparison of treatment efficacy between groups at various time points. Points are the mean areas of luciferase fluorescence for live intact animals in each group; error bars represent SEM (D). Tumor volumes measured on the last day of the experiment at autopsy using Vernier calipers and calculated using the formula $\mathrm{V}=(a \times b \times c) / 2(n=5)$ (E). Tumor weight of each group on the last day of the experiment $(n=5)(\mathrm{F})$. (G-J), Brusatol alone or in combination with chemotherapeutic agents markedly inhibited the tumor growth in Capan-2 orthotopic xenograft mouse model. Procedures for sequential whole-body imaging and quantification of tumor volume and weight were similar to those described in the $\mathrm{C}-\mathrm{F}$. ${ }^{*} P<0.05$ vs control and $\# P<0.05$ vs chemotherapeutic agent treatment alone. 
inhibit the proliferation of PanCa cells without affecting the viability of normal gastric epithelial cell. Besides, the anti-PanCa effect of brusatol was more potent than that of GEM or 5-FU alone, which also displayed great cytotoxic effect to normal gastric epithelial cell. It was also observed that brusatol showed a synergistic antiproliferative effect toward both PANC-1 and Capan-2 cell lines when combined with GEM or 5-FU, with the CI values being in the range of $0.2-0.8$, indicative of a strong synergistic action. Furthermore, it was found that brusatol monotherapy and combination treatment caused cell cycle arrest at G2/M phase, induced apoptosis, and inhibited several transcription factors and various biomarkers linked to survival, proliferation, and metastasis such as NF- $\kappa \mathrm{B}$ p65, Bcl-xL, and PCNA in PanCa cells. These results suggest that brusatol could accentuate the apoptotic effect of GEM or 5-FU by inhibiting anti-apoptotic proteins in PanCa cells. The observed down-regulation of antiapoptotic proteins was believed to contribute to the synergistic inhibition of PanCa cell growth exerted by the combined treatment of brusatol and GEM or 5-FU.

Based on these promising in vitro results, we therefore sought to evaluate the in vivo efficacy of brusatol in PanCa growth, using orthotopic models of PanCa derived from the highly malignant PANC-1 and Capan-2 cell lines. Our results suggested that daily brusatol administration for 28 days significantly decreased pancreatic tumor growth in both PANC-1 and Capan-2 cell orthotopic mouse models, without causing significant weight loss, mortality or other noticeable abnormalities on the heart, liver and kidney of the experimental animals. Furthermore, it was also shown that brusatol significantly synergized with GEM/5-FU and inhibited the tumor growth (tumor size, tumor weight and Lucsignal intensity) of mice more significantly than treatment with either single agent, while caused no observable side effects, further supporting the in vitro anti-PanCa activity. These findings strongly indicated that the enhanced anticancer efficacy and reduced cytotoxicity could be achieved through optimized combination treatment.

One of the major culprits involved in the development of drug resistance and prognosis of the disease is EMT, which refers to the absence of epithelial phenotype and presence of mesenchymal characteristics. The activation of EMT process is reported to facilitate embryonic development, tissue formation as well as tumor invasion and metastasis [19]. The hallmark of EMT is the loss of the epithelial homotypic adhesion molecule E-cadherin and the gain of mesenchymal markers such as vimentin [20]. In various human cancers, E-cadherin loss is related to poor prognosis, tumor progression and metastasis. Twist is a basic helix-loop-helix (basic Helix-loop-Helix bHLH) transcription factor located in autosomes. Several reports have indicated that Twist
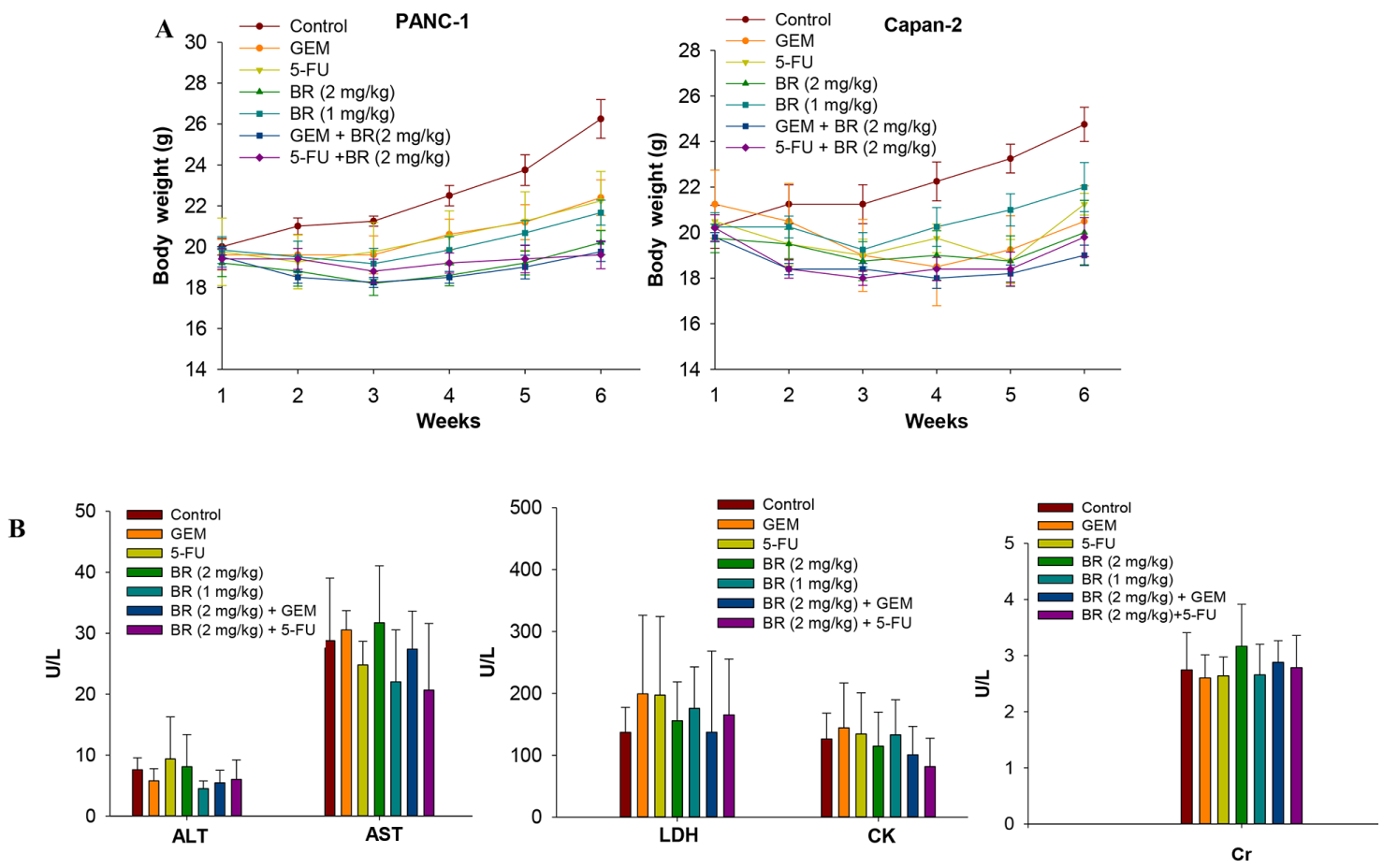

Figure 6: In vivo toxicity test of brusatol. Effects of brusatol alone or in combination with chemotherapeutic agents on the body weight of PANC-1 and Capan-2 orthotopic xenograft nude mice within 6 weeks (A), and plasma enzyme levels of alanine aminotransferase (ALT), aspartate aminotransferase (AST), lactate dehydrogenase (LDH), creatine kinase (CK) and creatinine (Cr) in Capan-2 orthotopic xenograft nude mice after treatment for 28 consecutive days (B). 
is the key regulator of the EMT process associated with tumor cell proliferation, differentiation, metastasis, invasion and anti-apoptotic process, with clinical implication of poor prognosis and metastasis in many kinds of cancers, such as breast, esophageal, gastric and prostate cancer. Twist is also an inhibitor of apoptosis [21-24]. Over expression of Twist often promotes cell colony formation, prevents apoptosis, and induces drug resistance and metastasis of tumor, with a consequence of an increased tumor invasiveness and poor prognosis [25]. In our experiments, brusatol monotherapy or combination treatment significantly increased the expression of E-cadherin and suppressed the expression of vimentin and Twist in both PANC-1 and Capan-2 cells, and the observation was believed to be associated with the inhibition of PanCa growth in vitro and in vivo.

This is the first report to show that brusatol was able to suppress PanCa growth and enhance the antiPanCa effect of GEM and 5-FU in an orthotopic mouse model. Based on our promising in vitro and in vivo results, brusatol could greatly sensitize PanCa to GEM and 5-FU while it exhibited a more favorable safety profile. The result indicated that brusatol might reduce the dose of chemotherapeutic agents while exerting superior beneficial effect for the treatment of PanCa. The synergistic inhibition by brusatol and chemotherapeutic agents observed both in vitro and in vivo, together with the absence of overt toxicity, strongly indicated that brusatol is a promising adjuvant to current chemotherapy regimen for this deadly human malignancy. However, to corroborate this potential, further experiments should be performed on this model to monitor the mouse survival benefit treated with the combinations.

Taken together, our present work laid a solid foundation for further in-depth studies to evaluate the overall survival benefit, long-term safety, pharmacokinetics in animal models including the genetically engineered pancreatic cancer mouse model. The results from this work also provided justification for conducting clinical trials in future to evaluate the safety and effectiveness of this natural product on pancreatic cancer. The development of brusatol into an anti-PanCa adjuvant would add new therapeutic dimensions to the current limited approach in the management of this most deadly malignancy in human.
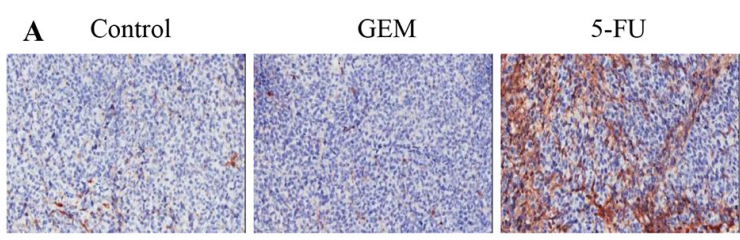

$\mathrm{BR}(2 \mathrm{mg} / \mathrm{kg})+\mathrm{GEM}$ BR $(2 \mathrm{mg} / \mathrm{kg})+5-\mathrm{FU}$

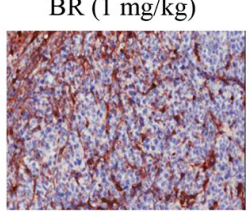

B Control

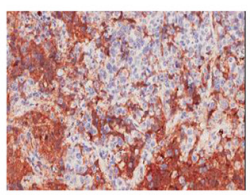

BR $(1 \mathrm{mg} / \mathrm{kg})$
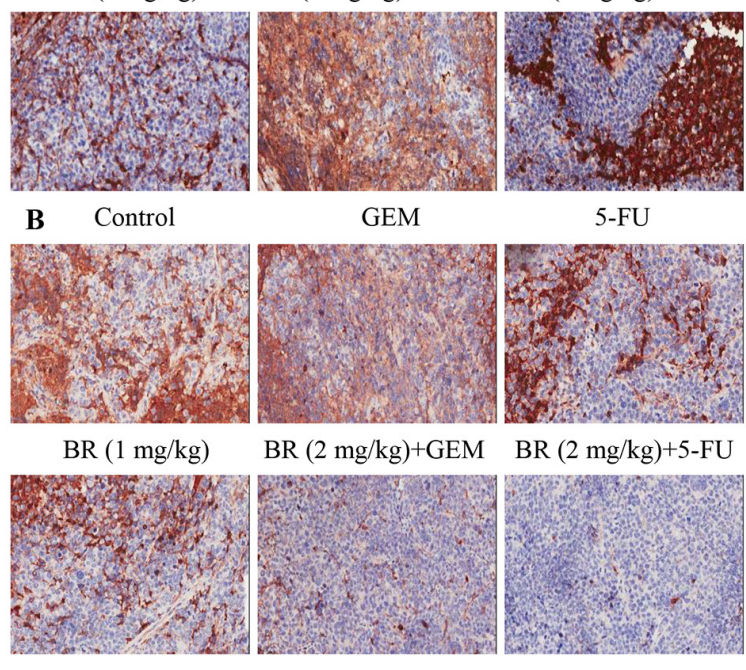

GEM

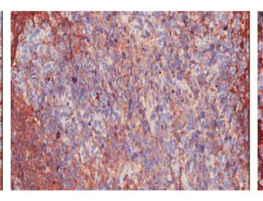

$5-\mathrm{FU}$

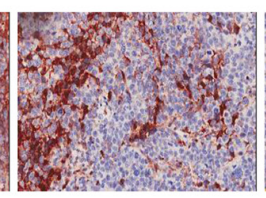

BR $(2 \mathrm{mg} / \mathrm{kg})+\mathrm{GEM}$

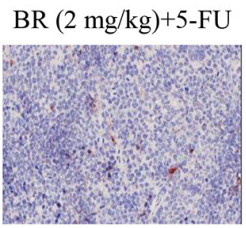

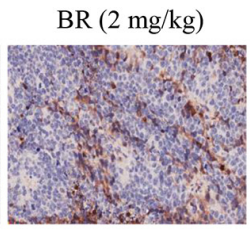

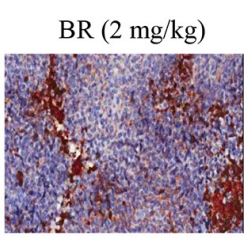

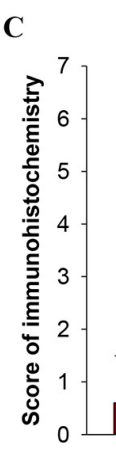

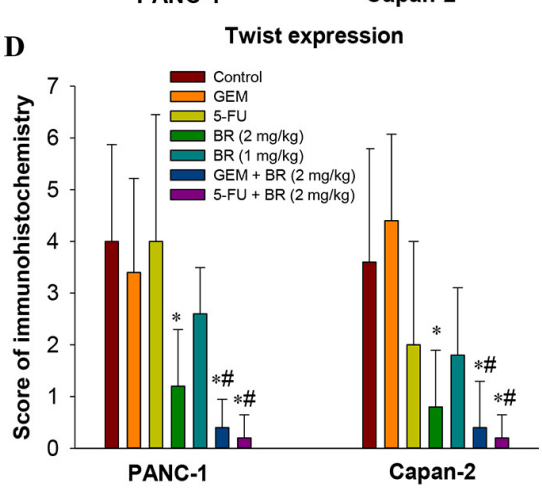

Figure 7: Expression of E-cadherin and Twist in the orthotopic xenograft tumor tissues by immunohistochemistry. (A-B) Effects of brusatol on E-cadherin (A) and Twist (B) expression levels in PANC-1 xenograft tissues. (C-D) Scoring of E-cadherin and Twist expression. Each slide was scored semi-quantitatively on the basis of percentage and intensity of the stained normal or neoplastic epithelial cells. The total score was the product of the scores for the intensity and positive rate of the staining. Samples analyzed were from 5 animals in each group. All images were taken at $200 \times$ magnification. The data in $\mathrm{C}$ and $\mathrm{D}$ are represented as the mean $\pm \mathrm{SEM} ; * P<0.05$ vs control and $\# P<0.05$ vs chemotherapeutic agent treatment alone. 


\section{MATERIALS AND METHODS}

\section{Cell lines and reagents}

Human pancreatic cancer cell lines (PANC-1, Capan-1, Capan-2 and SW1990) and non-tumorigenic human gastric epithelial cells were obtained from the ATCC (Manassas, VA, USA). The cell lines were authenticated by short-tandem repeat analysis. Cell lines were initially expanded and cryopreserved within 1 month of receipt and were typically used for 3 months; and at which time, a fresh vial of cryopreserved cells was used (2013-2015). These cells were maintained in culture as an adherent monolayer in DMEM or IMDM supplemented with $10 \%$ FBS.

Brusatol (CAS: 14907-98-3) was isolated from Bruceae Fructus in our laboratory, and its structural identity was confirmed by comparing its NMR and HRMS data with those published previously [12]. Its purity was determined to exceed $98 \%$ by HPLC analysis. All cell culture reagents were purchased from Invitrogen (Grand Island, NY, USA). Gemcitabine (GEM) (Gemzar, Eli Lilly, USA) and 5-Fluorouracil injection (5-FU) (China Food and Drug Administration, China; approval number H12020959) were procured through the Pharmacy of the Clinical Center, Shenzhen People's Hospital, Guangdong Province, China.

\section{Cytotoxicity assay, synergistic effects, cell death detection, apoptosis detection, cell-cycle analysis and plasma-specific enzyme level measurement}

The above assays were performed using kits from various manufacturers. Details are provided in the Supplementary Methods.

\section{Western immunoblotting and real-time PCR}

Western immunoblotting technique was used to analyze the expression of Twist, NF- $\mathrm{BB}, \mathrm{PCNA}, \mathrm{Bcl}-\mathrm{xl}$, vimentin and E-Cadherin. Real-time PCR technique was used to analyze the expression of Twist and E-Cadherin. Details are provided in the Supplementary Methods.

\section{Construction of PanCa cell lines stably expressing CMV-EGFP-linker-Luc}

To monitor the in vivo PanCa growth, PANC1 and Capan-2 cells were stably transfected with the luciferase gene. CMV-EGFP-linker-Luc-PGK-Puro-L.V. (constructed by Obio Technology, Shanghai, China) was a lenti-virus and could express green fluorescent protein and luciferase [26]. Lentiviral was transferred into PANC1 and Capan- 2 cells $\left(5 \times 10^{4}\right.$ cell/well in 24 -well plates $)$ for a $24 \mathrm{~h}$ infection in the presence of polybrene (final concentration $5 \mu \mathrm{g} / \mathrm{mL}$; Sigma-Aldrich). After $24 \mathrm{~h}$, the culture medium was then replaced by fresh medium. After culture for a further $72 \mathrm{~h}$, cells were incubated with puromycin (final concentration $2 \mu \mathrm{g} / \mathrm{mL}$ ) for a period of 14 days for selection. Expression of EGFP was observed by fluorescent microscopy and luciferase expression was confirmed using the Dual Luciferase Assay (Promega), and emitted light was directly proportional to the cell number.

\section{In vivo studies}

Male BALB/c nude mice (6 weeks of age) were supplied by the Laboratory Animal Services Centre, CUHK. Animals were bred and maintained in a pathogenfree condition with sterile food and water ad libitum in specifically designed air-controlled rooms with a 12-h light/dark cycle. The care and use of the animals were in compliance with the institutional guidelines, and the experimental procedures were approved by the Animal Experimentation Ethics Committee of CUHK (Ref. 14/086/MIS).

Human PanCa cell orthotopic xenograft nude mouse model was established using in situ injection method as described previously [27]. Capan-2 and PANC-1 cells, which were stably transfected with EGFP and luciferase as described above, were resuspended in PBS and kept on ice until injection. Male nude mice were anesthetized with ketamine/xylazine $(100 / 10 \mathrm{mg} / \mathrm{kg})$, a small left abdominal flank incision was made, and the pancreas was carefully exposed. Capan-2 $\left(2 \times 10^{6}\right.$ cells $)$ and PANC- 1 cells $(5 \times$ $10^{6}$ cells) were injected into the tail of the pancreas with a Hamilton syringe. The pancreas was then returned to the peritoneal cavity, and the abdominal wall and the skin were closed with 6-0 Dexon sutures. One week after tumor transplantation, mice were anesthetized and images of the tumors captured using a Carestream In Vivo Imaging System (MS FX PRO, USA). It was found that all tumors were of similar size. Mice were then randomized into 7 groups of 6 mice each: the control group, GEM group, 5-FU group, two brusatol treatment groups of different dosages (1 or $2 \mathrm{mg} / \mathrm{kg}$ ), GEM and brusatol $(2 \mathrm{mg} / \mathrm{kg}$ ) combination group, and 5-FU and brusatol $(2 \mathrm{mg} / \mathrm{kg})$ combination group.

The tumor-bearing mice were periodically injected i.p. with D-luciferin (150 mg/kg, Life Technologies, USA) and anesthetized once a week. After D-luciferin injection (10 min), luminescence was measured using a Carestream In Vivo Imaging System (MS FX PRO, USA), which could be used to obtain X-ray and concurrent bioluminescence images. By combining bioluminescence imaging (BLI) with digital x-ray, the system's highly improved sensitivity allowed us to precisely locate, identify and monitor tumor morphological changes [27,28]. Quantitative analysis of the optical signal capture was carried out with the Carestream MI software v5.0.5.29 (Carestream Health, Inc., Woodbridge, CT, USA). Fluorescence intensity from subcutaneous 
and intra-peritoneal tumors was measured by creating an automatic ROI threshold to $30 \%$ of each tumor's maximum intensity and the mean intensity of the area was examined. Fluorescence intensities were normalized to the peak angle of detection, and real-time determination of tumor burden was done by quantifying fluorescent surface area [29]. Mice were imaged on 7, 14, 21, 28 and 35 days after tumor implantation. Treatment was continued for 5 weeks and animals were sacrificed 1 week later. Whole blood was obtained by cardiac puncture. Primary tumors in the pancreas were excised and the final tumor volume was measured as $\mathrm{V}=(a \times b \times c) / 2$, where $a$ indicates the length, $b$ the width, and $c$ the depth of tumor [30]. Half of the tumor tissue was formalin-fixed and paraffin-embedded for immuno-histochemistry and routine H\&E staining. The other half was snap frozen in liquid nitrogen and stored at $-80^{\circ} \mathrm{C}$.

\section{Histology and tissue analysis}

Formalin-fixed tissues were embedded in paraffin and cut into $6-\mu \mathrm{m}$ sections. Sections were evaluated by H\&E staining and immunohistochemical analysis using antibodies specific for E-cadherin and Twist. The stained TMA slides were assessed independently by two experienced pathologists in a blinded manner. Each slide was scored semiquantitatively on the basis of percentage and intensity of the stained normal or neoplastic epithelial cells. The percentages of stained cells were scored using previously described methods $[31,32]$. Details of histological analysis are provided in the Supplementary Methods.

\section{Statistical analysis}

Results were expressed as mean \pm SEM. Data were analyzed by the test or ANOVA and results were considered significant at $P<0.05$. A significant interaction was interpreted by a subsequent median effect principle of the Chou-Talalay method.

\section{ACKNOWLEDGMENTS}

The authors thank all colleagues in Dr. Lin's laboratory for critical discussions. The authors also thank the flow cytometry and confocal core facility in the School of Chinese Medicine, CUHK for their expert technical assistance.

\section{CONFLICTS OF INTEREST}

The authors declare they have no conflicts of interests.

\section{GRANT SUPPORT}

This study was supported by grants from the Hong Kong Scholars Program 2012 (No. 2012-050), the Natural
Science Foundation of China (No. 81272667) and the Research Grants Council of Hong Kong (No. 469912).

\section{REFERENCES}

1. Bond-Smith G, Banga N, Hammond TM, Imber CJ. Pancreatic adenocarcinoma. BMJ. 2012; 344:e2476.

2. Siegel R, Ma J, Zou Z, Jemal A. Cancer statistics, 2014. CA Cancer J Clin. 2014; 64:9-29.

3. World Health Organization. (Stewart BW, Wild CP editors).World Cancer Report 2014. http://www.iarc.fr/en/ publications/books/wcr/.

4. Vincent A, Herman J, Schulick R, Hruban RH, Goggins M. Pancreatic cancer. Lancet. 2011; 378:607-20.

5. Neesse A, Krug S, Gress TM, Tuveson DA, Michl P. Emerging concepts in pancreatic cancer medicine: targeting the tumor stroma. Onco Targets Ther. 2013; 7:33-43.

6. Castellanos E, Berlin J, Cardin DB. Current treatment options for pancreatic carcinoma. Curr Oncol Rep. 2011; 13:195-205.

7. Yin SY, Wei WC, Jian FY, Yang NS. Therapeutic applications of herbal medicines for cancer patients. Evid Based Complement Alternat Med. 2013; 2013:302426.

8. Lau ST, Lin ZX, Zhao M, Leung PS. Brucea javanica fruit induces cytotoxicity and apoptosis in pancreatic adenocarcinoma cell lines. Phytother Res. 2008; 22:477-86.

9. Lau ST, Lin ZX, Leung PS. Role of reactive oxygen species in brucein D-mediated p38-mitogen-activated protein kinase and nuclear factor-kappaB signalling pathways in human pancreatic adenocarcinoma cells. Br J Cancer. 2010; 102:583-93.

10. Lau ST, Lin ZX, Liao Y, Zhao M, Cheng CH, Leung PS. Bruceine D induces apoptosis in pancreatic adenocarcinoma cell line PANC-1 through the activation of p38-mitogen activated protein kinase. Cancer Lett. 2009; 281:42-52.

11. Zhao M, Lau ST, Leung PS, Che CT, Lin ZX. Seven quassinoids from Fructus Bruceae with cytotoxic effects on pancreatic adenocarcinoma cell lines. Phytother Res. 2011; 25:1796-800.

12. Ren D, Villeneuve NF, Jiang $\mathrm{T}, \mathrm{Wu} \mathrm{T}$, Lau A, Toppin HA, Zhang DD. Brusatol enhances the efficacy of chemotherapy by inhibiting the Nrf2-mediated defense mechanism. Proc Natl Acad Sci USA. 2011; 108:1433-38.

13. Savagner P. The epithelial-mesenchymal transition (EMT) phenomenon. Ann Oncol. 2010 (Suppl 7); 21:vii89-92.

14. Neureiter D, Jäger T, Ocker M, Kiesslich T. Epigenetics and pancreatic cancer: pathophysiology and novel treatment aspects. World J Gastroenterol. 2014; 20:7830-48.

15. Chou TC, Motzer RJ, Tong Y, Bosl GJ. Computerized quantitation of synergism and antagonism of taxol, topotecan, and cisplatin against human teratocarcinoma cell growth: a rational approach to clinical protocol design. $\mathrm{J}$ Natl Cancer Inst. 1994; 86:1517-24. 
16. Chou TC. Theoretical basis, experimental design, and computerized simulation of synergism and antagonism in drug combination studies. Pharmacol Rev. 2006; 58:621-81.

17. Li Y, Ahmed F, Ali S, Philip PA, Kucuk O, Sarkar FH. Inactivation of nuclear factor kappaB by soy isoflavone genistein contributes to increased apoptosis induced by chemotherapeutic agents in human cancer cells. Cancer Res. 2005; 65:6934-42.

18. Damaraju VL, Bouffard DY, Wong CK, Clarke ML, Mackey JR, Leblond L, Cass CE, Grey M, Gourdeau H. Synergistic activity of troxacitabine (Troxatyl) and gemcitabine in pancreatic cancer. BMC Cancer. 2007; 7:121-32.

19. Son H, Moon A. Epithelial-mesenchymal transition and cell invasion. Toxicol Res. 2010; 26:245-52.

20. Chao YL, Shepard CR, Wells A. Breast carcinoma cells re-express E-cadherin during mesenchymal to epithelial reverting transition. Mol Cancer. 2010; 9:179-97.

21. Yang J, Mani SA, Donaher JL, Ramaswamy S, Itzykson RA, Come C, Savagner P, Gitelman I, Richardson A, Weinberg RA. Twist, a master regulator of morphogenesis, plays an essential role in tumor metastasis. Cell. 2004; 117:927-39.

22. Sasaki K, Natsugoe S, Ishigami S, Matsumoto M, Okumura H, Setoyama T, Uchikado Y, Kita Y, Tamotsu K, Sakamoto A, Owaki T, Aikou T. Significance of Twist expression and its association with E-cadherin in esophageal squamous cell carcinoma. J Exp Clin Cancer Res. 2009; 28:158-67.

23. Li QQ, Xu JD, Wang WJ, Cao XX, Chen Q, Tang F, Chen ZQ, Liu XP, Xu ZD. Twist1-mediated adriamycin-induced epithelial-mesenchymal transition relates to multidrug resistance and invasive potential in breast cancer cells. Clin Cancer Res. 2009; 15:2657-65.

24. Yang J, Weinberg RA. Epithelial-mesenchymal transition: at the crossroads of development and tumor metastasis. Dev Cell. 2008; 14:818-29.

25. Wang N, Guo D, Zhao YY, Dong CY, Liu XY, Yang BX, Wang SW, Wang L, Liu QG, Ren Q, Lin YM, Ma XT.
TWIST-1 promotes cell growth, drug resistance and progenitor clonogenic capacities in myeloid leukemia and is a novel poor prognostic factor in acute myeloid leukemia. Oncotarget. 2015; 6:20977-92. https://doi.org/10.18632/ oncotarget.4007.

26. Weber K, Mock U, Petrowitz B, Bartsch U, Fehse B. Lentiviral gene ontology (LeGO) vectors equipped with novel drug-selectable fluorescent proteins: new building blocks for cell marking and multi-gene analysis. Gene Ther. 2010; 17:511-20.

27. Pizzonia J, Holmberg J, Orton S, Alvero A, Viteri O, McLaughlin W, Feke G, Mor G. Multimodality animal rotation imaging system (Mars) for in vivo detection of intraperitoneal tumors. Am J Reprod Immunol. 2012; 67:84-90.

28. Lee SJ, Krauthauser C, Maduskuie V, Fawcett PT, Olson JM, Rajasekaran SA. Curcumin-induced HDAC inhibition and attenuation of medulloblastoma growth in vitro and in vivo. BMC Cancer. 2011; 11:144-57.

29. Phillips PA, Dudeja V, McCarroll JA, Borja-Cacho D, Dawra RK, Grizzle WE, Vickers SM, Saluja AK. Triptolide induces pancreatic cancer cell death via inhibition of heat shock protein 70. Cancer Res. 2007; 67:9407-16.

30. Li L, Yue GG, Lau CB, Sun H, Fung KP, Leung PC, Han Q, Leung PS. Eriocalyxin B induces apoptosis and cell cycle arrest in pancreatic adenocarcinoma cells through caspaseand p53-dependent pathways. Toxicol Appl Pharmacol. 2012; 262:80-90.

31. He C, Jiang H, Geng S, Sheng H, Shen X, Zhang X, Zhu S, Chen X, Yang C, Gao H. Expression of c-Myc and Fas correlates with perineural invasion of pancreatic cancer. Int J Clin Exp Pathol. 2012; 5:339-46.

32. Ohuchida K, Mizumoto K, Ishikawa N, Fujii K, Konomi H, Nagai E, Yamaguchi K, Tsuneyoshi M, Tanaka M. The role of S100A6 in pancreatic cancer development and its clinical implication as a diagnostic marker and therapeutic target. Clin Cancer Res. 2005; 11:7785-93. 